\title{
Theorie und Praxis erfolgreicher Blended Learning-Konzepte
}

\author{
Ulrike Tippe, Bertil Haack
}

\section{Ausgangssituation}

\subsection{Weiterbildungsbedarf und wirtschaftliche Grenzen}

In nahezu allen beruflichen Bereichen wächst der Bedarf an hoch qualifiziertem Fachpersonal. Als Beispiele seien die Begutachtung von Kfz-Schäden etwa nach Unfällen und die medizinische und pflegerische Versorgung älterer Menschen genannt: Aufgrund entsprechender gesetzlicher Anforderungen müssen Schadensgutachter mehrfach pro Jahr an Pflichtweiterbildungen teilnehmen. Ebenfalls aufgrund gesetzlicher Anforderungen sowie infolge der demografischen Entwicklung wächst der Bedarf an Qualifizierungsmaßnahmen für Mediziner und Pflegekräfte enorm. Beispielsweise werden allein im Bundesland Brandenburg bis zum Jahr 2010 insgesamt ca. 5.200 zusätzliche Fachkräfte im Bereich der Altenpflege benötigt [MASGF Brandenburg 2005, 77].

In allen diesen Fällen wird die berufliche Weiterbildung im gleichzeitig enger werdenden wirtschaftlichen Rahmen immer schwieriger und ist aufgrund des großen organisatorischen und finanziellen Aufwandes kaum mehr auf traditionellem Wege realisierbar. Als Ausweg bietet sich der Einsatz von Blended Learning-Lösungen an, in denen traditionelle Lehr- und Lernformen einerseits und eLearning mit Hilfe geeigneter elektronischer Werkzeuge andererseits sinnvoll kombiniert werden. (Als eLearning werden im hiesigen Kontext alle internetbasierten Lernund Qualifizierungsmaßnahmen verstanden, wobei die eLearning-Module auch als WBTs, d.h. als Web Based Trainings bezeichnet werden.)

\subsection{Blended Learning als mögliche Antwort}

Um den dargelegten Qualifizierungsbedarf bewältigen zu können, müssen innovative Aus- und Weiterbildungswege gefunden werden. Die Lehr- und Lernformen in der Ausund Weiterbildung sind organisatorisch wie inhaltlich anzupassen. Insbesondere ist nach modernen Wegen und Plattformen für die beruflichen Qualifizierungsmaßnahmen zu suchen und diese sind einzusetzen (vgl. hier und im Folgenden [Arnold 2004] und [Niegemann 2004] sowie [Baumgartner 2003]).

Lösungswege hierfür zeigen sich beispielsweise im Bereich von Fernstudiengängen an Universitäten und Fachhochschulen. Dort werden Phasen des Selbstlernens mit Präsenzunterricht kombiniert, wobei besonders das Selbstlernen durch geeignete eLearning-Module unterstützt wird.

Obwohl hohe Erwartungen an das eLearning gestellt wurden, hat es in seiner „Reinform“ nicht den durchschlagenden Erfolg gehabt: Fördert doch der völlige Verzicht auf den direkten Kontakt zum Dozenten in vielen Fällen nicht den Lernerfolg bzw. fehlt doch den Lernenden auch häufig der zwischenmenschliche Kontakt zu anderen Kursteilnehmern. Aber auch der finanzielle Aufwand für die Erstellung von eLearning-Modulen ist beträchtlich: Im Falle professionell entwickelten eLearning-Contents werden für eine Stunde Kurslaufzeit (das ist die Zeit, die ein WBT benötigt, um alle Lernsequenzen durchzuspielen, ohne individuelle Pausen, Lern- und Bearbeitungszeiten usw. zu berücksichtigen) je nach Erstellungsaufwand Preise zwischen $10.000 €$ bis hin zu $60.000 €$ berechnet.

Die beschriebenen Nachteile führten nach einem anfänglichen eLearning-Hype zu einer ersten Ernüchterung, der mittlerweile eine Konsolidierungsphase folgt. So hat sich in der letzten Zeit der Begriff des „Blended Learning“ eingebürgert. Dieser beschreibt ein Lernarrangement, das alle vorhandenen Lernformen (Präsenzseminare, Bücher, eLearning-Module) so miteinander kombiniert, dass der Lernprozess bestmöglich durchgeführt werden kann. Insbesondere macht die Kombination von Präsenzseminaren und eLearning-Angeboten aus mehreren pädagogischen Gesichtspunkten Sinn und erweist sich gegenüber reinem eLearning als Erfolg versprechender.

Somit geht es in der Praxis darum, Lernkonzepte zu entwickeln, die diese Aspekte berücksichtigen. Dabei ist einerseits festzustellen, dass es in zahlreichen Fachgebieten gut ausgearbeitete Präsenzseminare gibt und dass mittlerweile ausgefeilte, leicht erlernbare Werkzeuge zur Erstellung von eLearning-Modulen existieren („Rapid eLearning"). Andererseits fehlt es jedoch vielerorts nach wie vor an didaktisch hochwertigen eLearning-Modulen bzw. entsprechendem Know-how und entsprechender Unterstützung für die Entwicklung derartiger eLearningBausteine. Die Frage nach erfolgreichem Blended Learning muss also an diesem zuletzt genannten Faktum ansetzen und praktikable Ansätze bereitstellen, die insbesondere didaktische Erwägungen einbeziehen.

\subsection{Wege zu erfolgreichem Blended Learning}

Das vorliegende Papier widmet sich der grundsätzlichen Frage, wie Blended Learning erfolgreich gestaltet werden kann. Dabei nimmt es einerseits auf theoretische Überlegungen Bezug und zeigt andererseits anhand ausgewählter Beispiele, wie die Theorie in Sinne einer guten Praxis genutzt und umgesetzt werden kann.

Im Einzelnen wird zunächst dargelegt, was unter „erfolgreichem“ Blended Learning verstanden werden kann. Diese Überlegungen führen zu einem Vierklang aus Inhalt, Technik, Pädagogik/Didaktik und Soziologie als Erfolgsfaktoren für Blended Learning sowie zu je einer abstrakten Prozess- und Objektsicht auf das Blended Learning (Kapitel 2). Hiervon ausgehend wird durch Bezugnahme auf zwei Blended Learning-Projekte der Autoren gezeigt, wie die Objektsicht sinnvoll konkretisiert und für die Praxis handhabbar gestaltet werden kann (Kapitel 3). Danach 
werden Ergebnisse der Anwendung dieser Objektsicht in den angesprochenen Projekten vorgelegt (Kapitel 4). Zusammen mit den vorangehenden Überlegungen führen sie zu einer abschließenden Bewertung, inwieweit erfolgreiches Blended Learning möglich ist (Kapitel 5).

\section{Konzeptionelle Ansätze zur Entwick- lung, Bereitstellung und Nutzung von Blended Learning-Lösungen}

\subsection{Wann ist eine Blended Learning-Lösung ei- gentlich erfolgreich?}

Nachdem Blended Learning offensichtlich kein Selbstgänger ist, stellt sich Frage wie es „möglichst erfolgreich“ gestaltet und vor allem was unter "möglichst erfolgreich" verstanden werden kann. Bei der Beantwortung kommt es auf die Perspektive an, aus der diese Frage betrachtet wird. Beispielsweise heißt „erfolgreich“ aus Sicht des Lernenden, dass er mit Hilfe des Blended Learning-Angebotes einen auf ihn zurecht geschnittenen und damit für ihn angemessenen Weg durch das zu lernende Themengebiet finden und sich dieses effektiv und effizient erschließen kann. Aus Sicht des Anbieters von Blended Learning-Lösungen bedeutet „erfolgreich“ natürlich auch, dass der Lerner mit dem Bildungsangebot im eben genannten Sinne zurecht kommt und zufrieden ist. Spätestens dann aber, wenn der Anbieter auch die Aufgabe der Entwicklung von Blended Learning-Kursen wahrnimmt, besitzt „erfolgreich“ mindestens eine weitere Facette. Jetzt kommt es ebenso darauf an, die Entwicklung verschiedener Blended Learning-Pakete mit unterschiedlichen Lerninhalten möglichst effektiv und effizient zu gestalten.

Insgesamt führen alle verschiedenen Sichten auf „möglichst erfolgreiche“ Blended Learning-Lösungen zur Frage nach sinnvollen Konzepten für die effektive und effiziente Entwicklung, Bereitstellung und Nutzung entsprechender Blended Learning-Angebote. Diese Aspekte betreffen einerseits die Prozesse eben der Entwicklung, Bereitstellung und Nutzung einer Blended Learning-Lösung sowie andererseits das Artefakt „Blended Learning-Lösung“ selbst in seiner Eigenschaft als Operationsobjekt (Produkt bzw. Handlungsgegenstand) dieser Prozesse. Wie bereits eingangs angedeutet wurde, muss hierbei die Sicht des Lerners als ausschlaggebend eingeschätzt und daher vor allen anderen Bezügen berücksichtigt werden: Nur dann, wenn der Lerner erfolgreich mit dem Artefakt lernen kann, haben sich die Entwicklung, Bereitstellung und Nutzung des Artefaktes gelohnt. Anders ausgedrückt: Didaktische Überlegungen müssen als Basis für die in Rede stehenden Prozesse und das Artefakt Blended Learning-Lösung dienen. Umgekehrt dürfen diese aber auch nicht losgelöst von anderen Betrachtungsebenen gesehen werden: Natürlich spielen beispielsweise auch die Lerninhalte und das Miteinander im Lernprozess eine wesentliche Rolle.

\subsection{Der Vierklang aus Inhalt, Technik, Pädagogik/ Didaktik und Soziologie als Erfolgsfaktor}

Um erfolgreich eLearning-Module zu entwickeln und in ein Blended-Learning-Konzept zu integrieren, ist $\mathrm{zu}$ vergegenwärtigen, dass hierbei neben dem Content im
Wesentlichen drei weitere Bereiche zu berücksichtigen sind. Es handelt sich prinzipiell um

- inhaltliche,

- technische,

- pädagogisch/didaktische sowie

- soziologische

Aspekte, die alle miteinander verbunden bzw. voneinander abhängig sind und damit gemeinsam in einem aufeinander abgestimmten harmonischen Vierklang zu beachten sind. Die folgende Abbildung verdeutlicht die relevanten Themen und Schlagworte sowie deren Zusammenhänge:

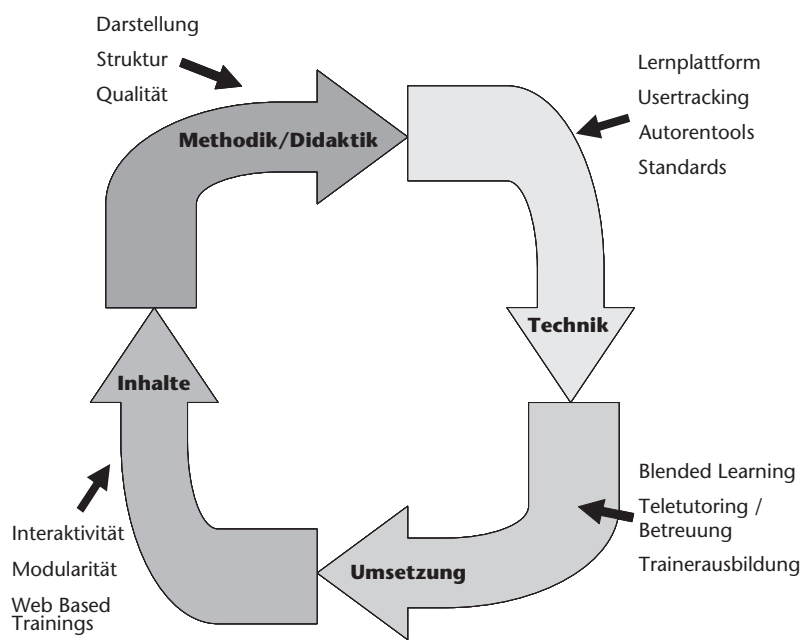

Abb. 1: Betrachtungsebenen für Blended Learning-Konzepte

\subsection{Prozesssicht: Ein Vorgehensmodell für Entwicklung, Einsatz und Anwendung von eLearning-Komponenten}

Analog zur Entwicklung und zum Einsatz „normaler“ Software muss ein geeignetes Vorgehensmodell Basis jedweder multimedial gestützten Aus- oder Weiterbildung sein. Mit seiner Hilfe werden alle Schritte zur Entwicklung und Durchführung des Qualifizierungsangebotes koordiniert. Es wird so festgelegt, wer wann was zu tun hat beziehungsweise tun darf. Darüber hinaus enthält es Festlegungen über die dabei jeweils einzusetzenden Methoden und Werkzeuge sowie über die erwarteten Arbeitsergebnisse.

Die Entwicklung einer Blended Learning-Anwendung ist ein Projekt und kann als solches etwa mittels des folgenden, aus der Softwareentwicklung bekannten Vorgehensmodells organisiert werden (vgl. beispielsweise [Balzert 2000] sowie ergänzend [Pawlowski 2001]):

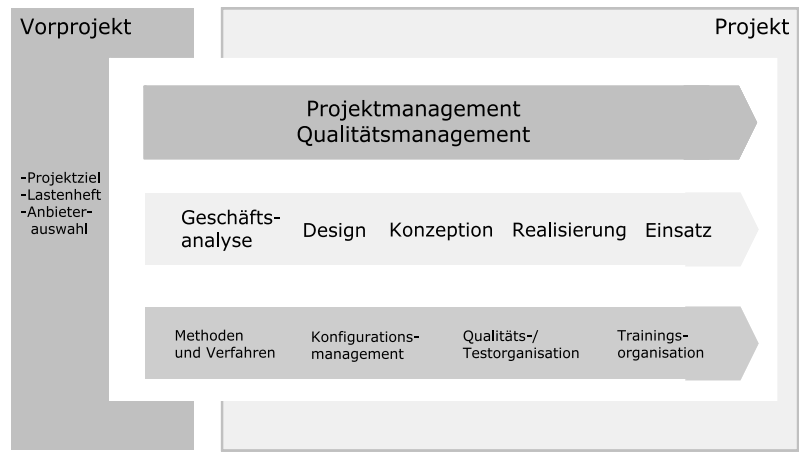

Abb. 2: Ein mögliches eLearning-Vorgehensmodell 
Kennzeichnend darin ist das Zusammenspiel von Leistungserbringungsprozess, Führungs- und Supportprozessen. Die Leistungserbringung erfolgt beispielsweise in den Phasen Geschäftsanalyse, Design, ..., Einsatz mit zugehörigen Meilensteinen und Arbeitsergebnissen. Sie wird mittels der Führungsprozesse Projekt- und Qualitätsmanagement geplant, durchgeführt, kontrolliert und gesteuert und erfährt innerhalb der Supportprozesse die notwendige Bereitstellung etwa einer Qualitäts- und Testorganisation.

\subsection{Objektsicht: Das Modell BLESS}

Die Prozesssicht muss nun noch durch eine geeignete Sicht auf das Operationsobjekt ergänzt werden. Dazu bietet sich die Blended Learning Systems Structure BLESS [Derntl / Motschnig-Pitrik 2005, S. 113ff.] an. Sie liefert ein Modell, nach dem der Aufbau eines eLearning-Systems gestaltet, verstanden, beschrieben und realisiert werden kann. Hier wird ebenso wie in der Prozesssicht von den Ähnlichkeiten und Übereinstimmungen in der Entwicklung, Bereitstellung und Nutzung von „normaler“ Anwendungssoftware und Blended Learning-Lösungen ausgegangen. Zusätzlich werden die für eLearning respektive Blended Learning als notwendig herausgestellten Betrachtungsebenen Fachinhalt (Lernstoff), Technik (Entwicklungs- und Lernplattform), Didaktik (Gestaltung des Lernangebotes) und Soziologie (Zusammenarbeit der Lehrenden und Lernenden) sowie deren Beziehungen zueinander berücksichtigt. Dieser „Vierklang“ wird durch Integration der einzelnen Aspekte in einem Schichtenmodell mit insgesamt sechs verschiedenen Ebenen erreicht:

- Layer 0: Learning Theory \& Didactic Baseline

Festlegung der umzusetzenden Lerntheorie und der didaktischen Ausrichtung des Kurses als „Treiber“ für alle weiteren Layer - heutzutage lauten die Stichworte Konstruktivismus und Handlungsorientierung

- Layer 1: Blended Learning Courses

Repräsentation des konkreten Blended Learning-Angebotes

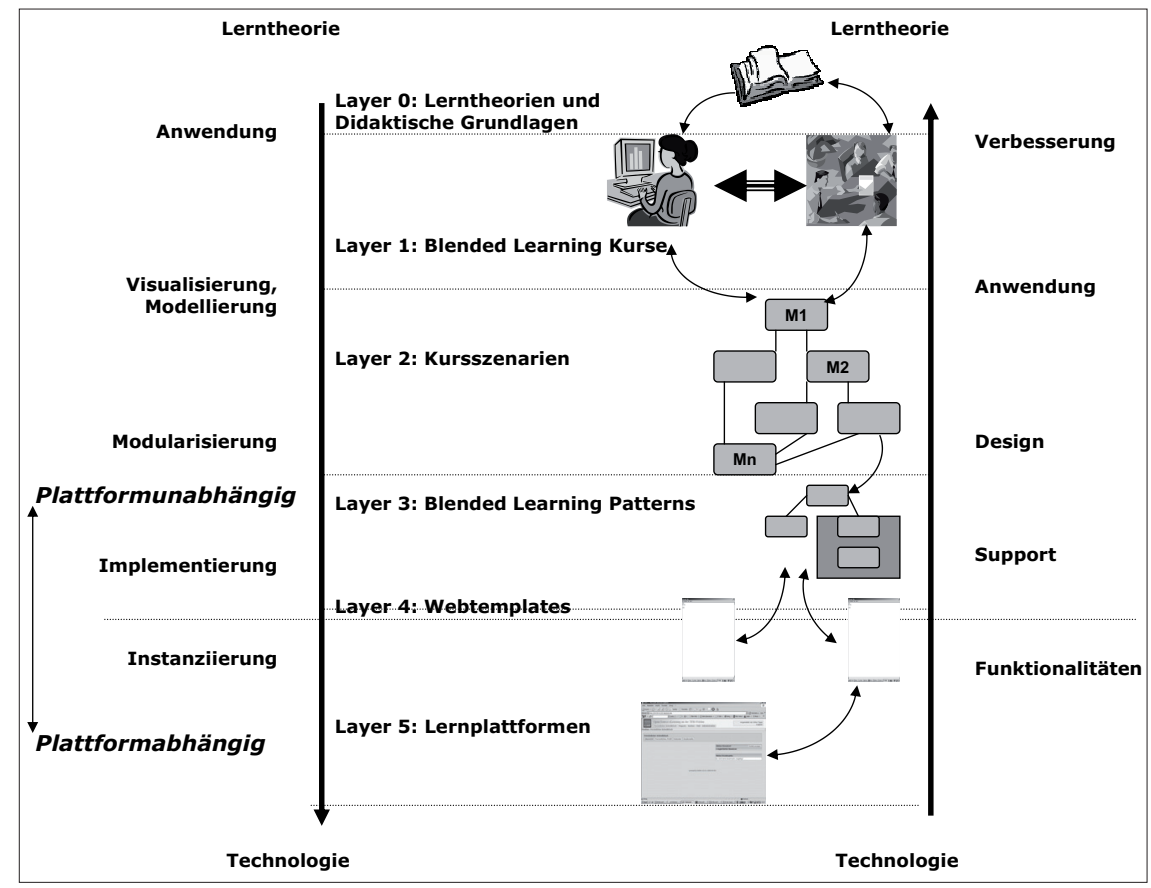

Abb. 3: Das BLESS-Modell [Derntl / Motschnig-Pitrik 2005]
- Layer 2: Course Scenarios - Learning Traces Denkbare, sinnvolle und mögliche Kursabfolgen

- Layer 3: Blended Learning Patterns - Learnflow Patterns Wiederkehrende Lernabläufe

- Layer 4: Templates - Learnflow Templates Vorgefertigte Vorlagen zur Nutzung innerhalb einer eLearning-Anwendung

- Layer 5: Learning Platform Technische Basis des eLearning-Angebotes

Die Zusammenhänge der Ebenen werden aus Abbildung 3 ersichtlich.

\subsection{Umsetzung in die Praxis}

In der Praxis kommt es nun darauf an, die skizzierten Konzepte so zu konkretisieren, dass sie als Grundlage für die Entwicklung, Bereitstellung und Nutzung von Blended Learning-Lösungen dienen können. Nachstehend werden die Charakteristika eines konkreten, in der praktischen Anwendung befindlichen BLESS-konformen Konzeptes für die Entwicklung, den Einsatz und die Anwendung von Blended Learning-Lösungen zusammengefasst.

\section{Konkretisierung der konzeptionellen Ansätze}

\subsection{Werkzeuge: Learning Management Systeme und Autorentools (Layer 5)}

Bezüglich der Werkzeuge („Technik“) ist grundsätzlich zwischen „Learning Management Systemen“ („Lernplattformen“) und „Autorenwerkzeugen“ zu unterscheiden. Vereinfacht ausgedrückt dienen Learning Management Systeme (LMS) vorrangig zur Verwaltung/Administration von Inhalten und Nutzern (Lerner, Moderatoren), wohingegen die Autorenwerkzeuge („Autorentools“) zur Erstellung der Module (Web Based Trainings, WBTs) gedacht sind. Oftmals sind in Learning Management Systemen bereits Autorentools integriert, die häufig jedoch hinsichtlich der Funktionalität bzw. der Verwendung multimedialer Komponenten eingeschränkt sind. Aus diesem Grunde werden separate $\mathrm{Au}$ torenwerkzeuge angeboten, die die Erstellung didaktisch hochwertiger eLearning-Module ermöglichen. Um diese Module erfolgreich in Learning Management Systeme integrieren zu können, haben sich in den letzten Jahren Standards etabliert (SCORM, AICC).

Sowohl Learning Management Systeme als auch Autorentools, die jeweils die üblichen Standards erfüllen, gibt es mittlerweile viele am Markt. Auch ihre Funktionalitäten bzw. Möglichkeiten der Integration von multimedialen Komponenten sind ähnlich, so dass der Fokus nun mehr auf die Erstellung von Inhalten, d.h. auf didaktische und pädagogische Aspekte gelegt werden 
sollte. Anders formuliert: Die Technik ist da, sie muss jetzt „nur noch“ optimal genutzt werden. (Die Autoren setzen einerseits das Open Source Learning Management System „ILIAS“ und andererseits das Autorentool „Dynamic Power Trainer" der Firma Dynamic Media ein.)

\subsection{Methoden: Learning Traces, Learnflow Pat- tern und Learnflow Templates (Layer 2-4)}

Im Gegensatz zur Technik stellt das methodische Vorgehen bei der Entwicklung von eLearning-Lösungen nach wie vor eine große Herausforderung dar. Grundsätzlich ist es zunächst durch die Erstellung von Drehbüchern und deren Umsetzung mittels Autorenwerkzeugen geprägt. Darüber hinausgehend kommen Learning Traces, Learnflow Pattern und Learnflow Templates zum Einsatz:

- Learning Traces

Learning Traces bieten die Möglichkeit, verschiedene Kursabfolgen („tracks“) im Rahmen eines eLearningAngebotes zu realisieren und dem Lernenden so die Auswahl des für ihn entsprechend seiner Vorkenntnisse bzw. Wünsche optimalen Kursangebotes zu gestatten.

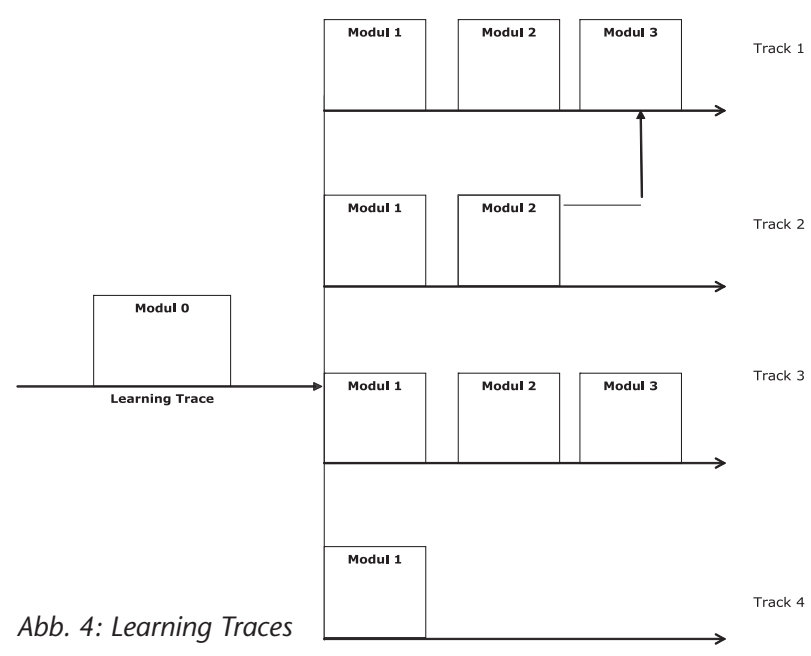

- Learnflow Pattern

Learnflow Pattern sind Lernmuster bzw. -abläufe, die in allen Lehr- und Lernprozessen wiederkehren und die die Umsetzung der je spezifischen Lernprozesse mittels geeigneter wiederkehrender Bausteine erlauben (vgl. [Derntl / Motschnig-Pitrik 2003]). Als Beispiele seien die Erarbeitung von Begriffen oder fachlichen Zusammenhängen durch den Lernenden sowie die Erledigung von Übungs- oder Prüfungsaufgaben genannt.

- Learnflow Templates

Learnflow Templates sind schließlich vorgefertigte abstrakte Bausteine einer eLearning-Anwendung. Sie realisieren Learnflow Pattern und können durch geeignetes „Customizing“ konkretisiert („instanziiert“) und auf die jeweilige eLearning-Anwendung zugeschnitten werden.

Durch dieses Vorgehen wird die Wiederverwendung der eLearning-Bausteine analog der Wiederverwendung von Software ermöglicht. Die entwickelten eLearningKomponenten können wie ein Gerüst in verschiedenen Themenbereichen mit jeweils anderen fachlichen Inhalten genutzt werden, indem beispielsweise jeweils eine Rahmengeschichte zugrunde gelegt wird, in die der Lernstoff und die Prüffragen eingebettet sind, und deren Inhalt, Kontext sowie die handelnden Personen je nach Lerninhalt ausgetauscht oder variiert werden.

\subsection{Lösung: Konzeption und Realisierung eines eLearning-Moduls (Layer 1-2)}

Bei der Konzeption und Realisierung eines eLearningModuls sind aus Sicht der Layer 1 und 2 mindestens die folgenden vier Aspekte zu beachten, die hier auch im Sinne von Handlungsempfehlungen verstanden werden sollen:

- Didaktische Qualität (Komplexität, „Merkbarkeit“) Hier kommt es ganz wesentlich darauf an, ein klares, differenziertes Lernangebot vorzulegen, das auf das Nötigste reduziert ist („Didaktische Reduktion“). Den Lernenden sollte es ermöglicht werden, ihr subjektives Wissen einzubringen, indem sie Neues mit bereits Erlerntem verknüpfen können. Dies schließt eine genaue Zielgruppenanalyse mit ein. Hinsichtlich der „Merkbarkeit" ist einerseits eine Orientierung an der „magischen Sieben" empfehlenswert: Eine Person kann sich bis zu sieben Begriffe, Bilder, Punkte o.ä. recht gut merken. Mehr Information auf einer Seite oder in einem Lernobjekt können nicht mehr so gut von den Lernenden verarbeitet werden. Andererseits sind Verknüpfungen von Neuem mit Bekanntem durch Visualisierung (passende Bilder / Animationen) und Wiederholung gut geeignet, um das Ziel, den Lernstoff in das Langzeitgedächtnis zu transportieren, zu erreichen.

- Struktur („Innere Ordnung“)

Insbesondere hinsichtlich der „Merkbarkeit“ und den damit notwendigen Wiederholungsmöglichkeiten des Lernstoffs ist eine klare modulare Struktur erforderlich. Innerhalb der Module sollten Inhalte, Zusammenfassungen, Zwischenfragen, Kommentare usw. stets an derselben Stelle und in gleichen Abständen erfolgen. Diese „logischen Strukturen“ dürfen nicht aufgebrochen, sondern müssen strikt eingehalten werden.

- Lerntyporientierung

Um Ganzheitliches zu praktizieren, ist das „Lernen mit allen Sinnen“ gefordert. Das heißt, dass Lernen über unterschiedliche Sinneskanäle (sensorische Kanäle) erfolgt. Hier wird zwischen auditiven, visuellen oder optischen und kinästhetischen oder handlungsorientierten Lerntypen unterschieden, wobei in der Regel Mischformen mit unterschiedlich stark ausgeprägten Schwerpunkten in die eine oder andere Richtung vorliegen. Grundsätzlich können folgende Gestaltungshinweise gegeben werden: Visualisierungen nutzen, Bilder und Texte müssen zusammenpassen, zusammenhängende Bilder und Worte müssen räumlich beieinander liegen und gleichzeitig präsentiert werden, Animationen gezielt einsetzen (bei „Anfängern" mehr, bei „Fortgeschrittenen“ weniger).

- Handlungsorientierung

Die Handlungskompetenz setzt sich aus den Komponenten „Fachkompetenz", „Methodenkompetenz" und "Sozialkompetenz" zusammen. Diese mit Hilfe von eLearning-Modulen auszubilden, ist mit Sicherheit ein anspruchsvolles Ziel. Auf den ersten Blick liegt es nahe, anzunehmen, dass mit Blended Learning lediglich Fachwissen angemessen vermittelt und damit nur die Fachkompetenz ausgebildet werden kann. Dennoch ist 
es möglich, die anderen Kompetenzfelder ebenfalls zu fördern. Ein Weg dazu besteht z.B. darin, den Lernstoff in eine Rahmenhandlung einzubinden, die aus dem Umfeld hervorgeht, aus dem der Lernende stammt, das er gut beurteilen kann und in dem er sich weitestgehend auskennt. Aus diesem Kontext heraus Inhalte zu vermitteln, Entscheidungen (Zwischenfragen) und den Aufbau neuer inhaltlicher Verknüpfungen abzuverlangen, also Handlungsprodukte als wesentliches Merkmal handlungsorientierten Unterrichts herstellen zu lassen [Jank/Meyer 1991, S. 356], führt weit über das reine Vermitteln von Inhalten/Fakten hinaus.

\section{Zwei Beispiele: Umsetzung der Theorie in die Praxis}

\subsection{Charakteristika des konkreten Blended Learning-Systems}

Ausgehend von obigen Überlegungen haben die Autoren zwischenzeitlich ein BLESS-konformes Blended LearningSystem konzipiert und prototypisch in zwei Anwendungsfällen zum Einsatz gebracht. Das System basiert auf der Lernplattform ILIAS und wurde mit dem Dynamic Power Trainer als Autorenwerkzeug realisiert. Es besitzt zusammengefasst folgende Merkmale:

- Ein flexibles Vorgehensmodell mit einer spezifischen Kombination von Präsenz- und Selbstlernanteilen je eLearning-Kurs,

- eine innovative Kombination von Learning Traces, Learnflow Pattern und Learnflow Templates,

- die Verbindung moderner Autoren- und Lernmanagementsysteme auf Basis aktueller Schnittstellen (Stichwort: SCORM) sowie

- Lernunterstützung durch den Einsatz von elektronischen Tutoren, Hotline-Support via Mail, Kommunikation in Chat-Rooms und Foren.

Aufgrund dieses flexiblen Lösungsdesigns ist das System universell im Rahmen von „Teleausbildungsmaßnahmen“ einsetzbar. Es kann durch das oben erwähnte Customizing - speziell: durch den Austausch des modular strukturierten Lernstoffs - relativ leicht in vielen Fachgebieten genutzt werden und damit einen Beitrag zur effektiven und effizienten Befriedigung des eingangs geschilderten Weiterbildungsbedarfs leisten. Konkret steht es zurzeit prototypisch für Weiterbildungsmaßnahmen im Rahmen der Qualifikation von Kfz-Schadensgutachtern sowie auf dem Gebiet der Schulung von Pflegekräften für den Einsatz in der Gerontopsychiatrie zur Verfügung.

\subsection{Weiterbildung von Kfz-Schadensgutachtern}

Kfz-Schadensgutachter sind gesetzlich verpflichtet, sich regelmäßig fortzubilden. Zukünftig ist geplant, einen Teil dieser Ausbildung via Blended Learning zu realisieren, um beispielsweise Reisekosten der Gutachter für die Fahrten zum Schulungsort einzusparen, aber auch um den Gutachtern die Möglichkeit zu bieten, die eigenen Lernzeiten und die eigene Lerngeschwindigkeit festzulegen. Hier exemplarisch drei Bildschirmansichten aus dieser, auf einer Rahmenhandlung basierenden Blended LearningLösung:

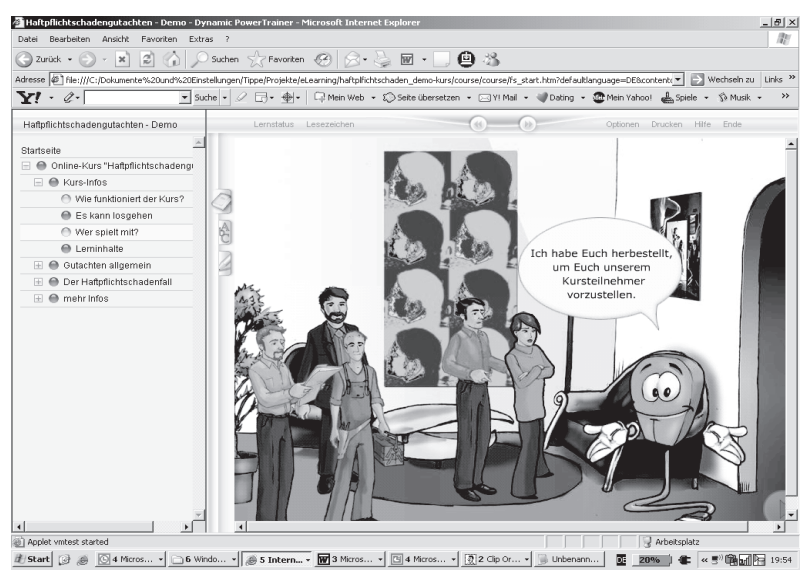

Abb. 5: Einführung in die Rahmenhandlung, Vorstellung der agierenden Personen

Der Avatar „Klaus, die Maus“ führt den Lernenden in die Rahmenhandlung (Verkehrsunfall) ein und stellt die handelnden Personen vor.

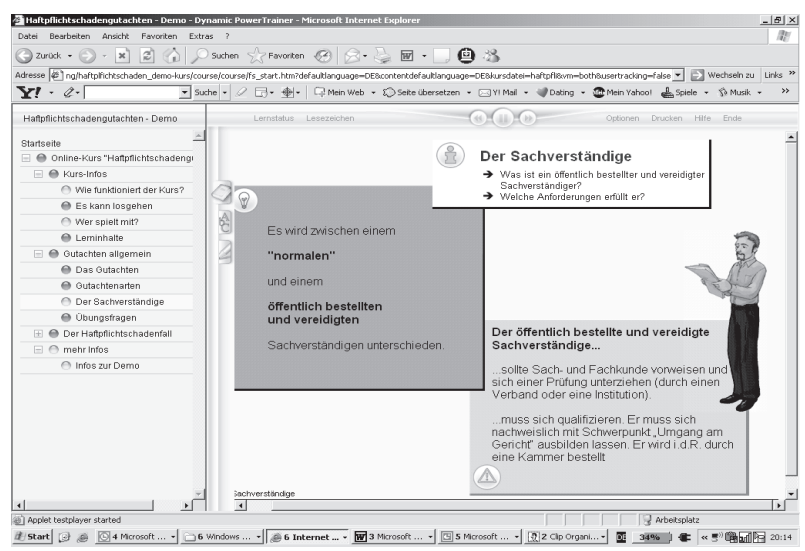

Abb. 6: Wissensvermittlung

Die Wissensvermittlung erfolgt in einer je gleichen Weise: Neben einem durch ein „i“ gekennzeichneten Informationsblock, in dem das behandelte Thema zusammengefasst und das eigentliche Lernziel formuliert werden, gibt es den Wissensblock sowie den Hinweisblock: Innerhalb des mit einer Glühlampe gekennzeichneten Wissensblocks werden die detaillierten Lerninhalte dargestellt. Im Hinweisblock (symbolisiert durch ein Ausrufungszeichen in einem Dreieck) stehen schließlich interessante Ergänzungen zu den Lerninhalten sowie Tipps zu den später zu beantwortenden Übungsfragen.

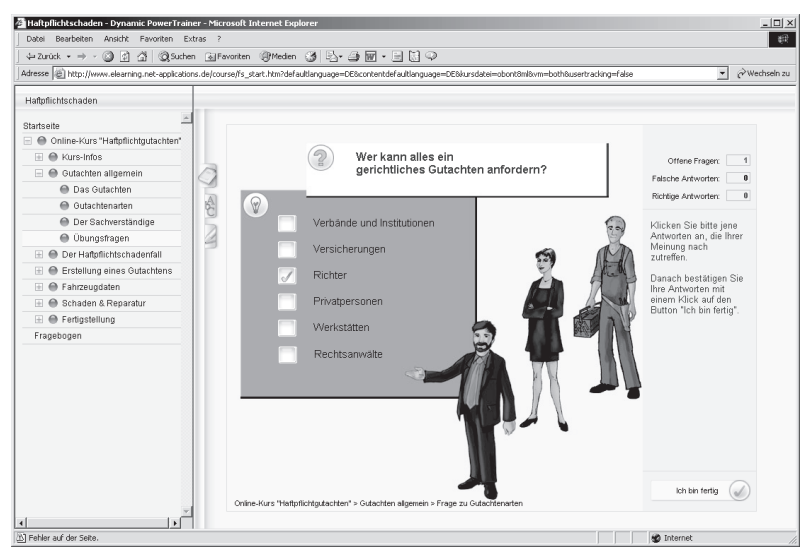

Abb. 7: Wissensüberprüfung 
Nach jeder Lerneinheit muss sich der Lernende den durch ein „?" gekennzeichneten Prüffragen unterziehen. Er kann so seinen Lernerfolg ermitteln und den weiteren Kursablauf daraus ableiten (z.B. Fortgang im Kurs bei fehlerfreier Beantwortung oder Rückschritt zu vorherigen Kursabschnitten bei fehlerhaften Antworten).

\subsection{Weiterbildung von Pflegekräften}

Im Pflegebereich befinden sich Ideen zur Umsetzung von eLearning-Maßnahmen zumindest teilweise noch in den „Startlöchern“, d.h. Blended-Learning-Konzepte haben bisher kaum oder gar nicht Eingang in die Aus- und Weiterbildung von Mitarbeitern gefunden. So steht eine vergleichbare Entwicklung beispielsweise im Falle gerontopsychiatrischer Pflegedienstleistungen noch aus. Entsprechende Lehr- und Lernwege müssen neu konzipiert werden. Dabei gilt es nicht nur, die jeweiligen fachlichen Fähigkeiten zu berücksichtigen und auszubilden, sondern auch den wachsenden Anforderungen an die Managementkompetenzen der Pflegekräfte gerecht zu werden. Dies ist zum Beispiel vor dem Hintergrund sich ausdifferenzierender Organisationsformen in der Gerontopsychiatrie vonnöten.

Ebenfalls von der Standard-Lösung ausgehend wurde bereits ein erster Prototyp für die Blended Learning basierte Weiterbildung von Pflegekräften im Bereich der Gerontopsychiatrie entwickelt. Hier die drei den vorangehenden Bildschirmansichten entsprechenden Screenshots aus diesem Prototyp. Sie zeigen die Verwandtschaft der verschiedenen eLearning-Lösungen, d.h. sie verdeutlichen, dass die einzelnen Lösungen durch Customizing eines vorgegebenen Standards entstanden sind:

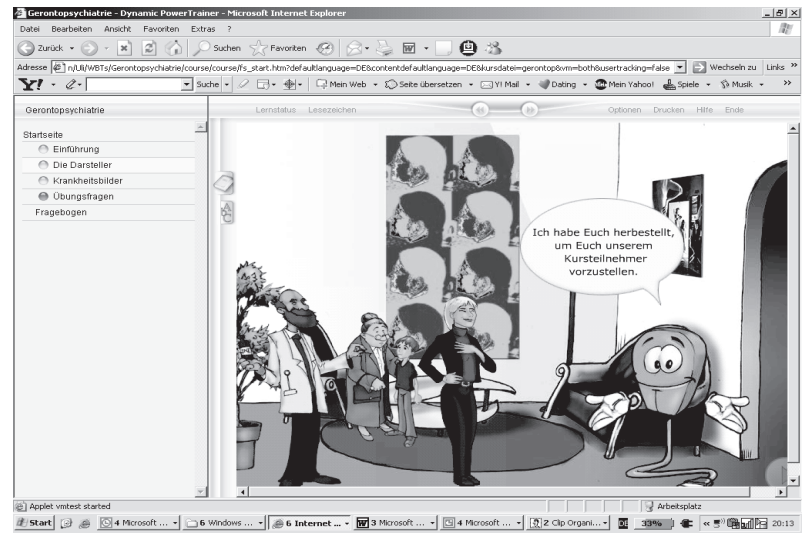

Abb. 8: Einführung in die Rahmenhandlung, Vorstellung der agierenden Personen

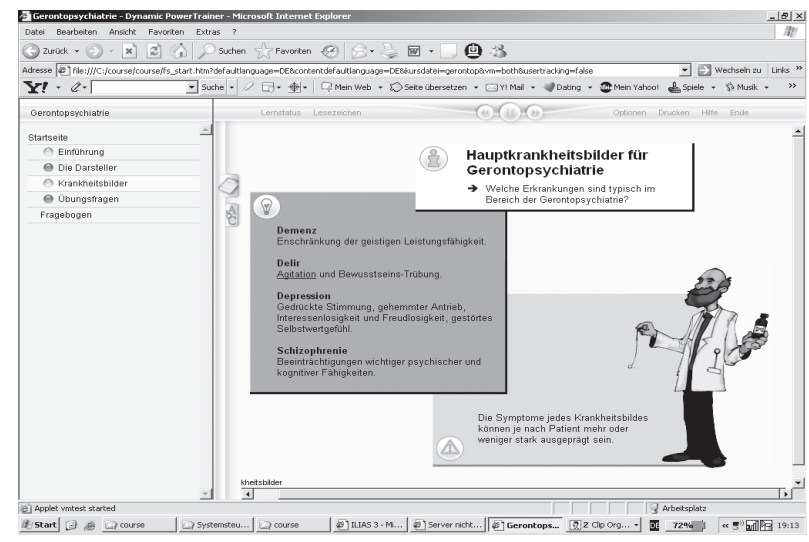

Abb. 9: Wissensvermittlung

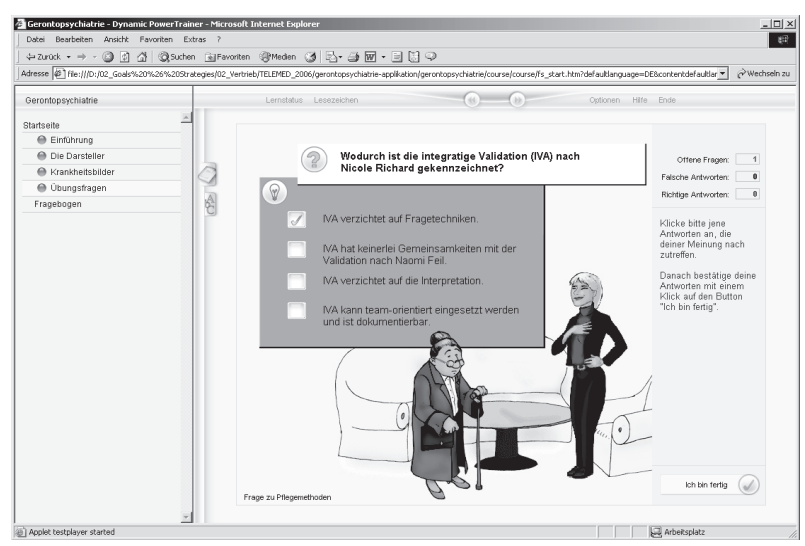

Abb. 10: Wissensüberprüfung

Insgesamt wird deutlich, dass bei beiden Entwicklungen nach ein und demselben Schema vorgegangen wurde. Der Einsatz von Learnflow Patterns und Learnflow Templates (BLESS-Layer 3 und 4) hat die Entwicklung im Rahmen der Projekte nicht nur vereinfacht sondern insbesondere auch wirtschaftlicher gemacht. Den Autoren liegen allerdings noch keine gesicherten Erkenntnisse bzw. Kalkulationen vor, ob diese Ergebnisse auf andere Projekte übertragbar sind. Die bisherigen Erfahrungen stimmen jedoch zuversichtlich.

\section{Abschließende Bewertung}

Qualifizierungsmaßnahmen mittels des präsentierten Blended Learning Systems tragen in stärkerem Maße als tradierte Weiterbildungsaktivitäten den wachsenden betriebswirtschaftlichen Anforderungen an entsprechende Maßnahmen in verschiedenen Fachgebieten wie z. B. Medizin und Pflege Rechnung. Beispielsweise erfordern sie keine komplette Freistellung der Teilnehmenden von ihrer Arbeit, verringern Reisekosten und -zeiten und reduzieren damit die Ausfallzeiten und -kosten für den jeweiligen Arbeitgeber. Insofern ist dieses Konzept ebenso für niedergelassene Ärzte wie für Pflegekräfte, kleinere und mittlere Unternehmen, Ingenieurbüros, Gutachter etc. geeignet, die sich regelmäßig fortbilden müssen, deren berufliche und / oder wirtschaftliche Situation ihnen aber erhebliche Grenzen für diese Weiterbildungsmaßnahmen setzt.

Darüber hinaus stellt eine Konzeption von Blended Learning-Kursen nach dem BLESS-Modell die Weichen für eine perspektivisch günstigere Entwicklung qualitativ hochwertiger eLearning-Module. 


\section{Literatur}

Patricia Arnold u.a.: „E-Learning, Handbuch für Hochschulen und Bildungszentren“. BW Bildung und Wissen, 2004

Helmut Balzert: „Lehrbuch der Software-Technik“. Spektrum Akademischer Verlag, 2000

Peter Baumgartner: „Didaktik, eLearning-Strategien, Softwarewerkzeuge und Standards- Wie passt das zusammen?" Erschienen in Maike Franzen: „Mensch und E-Learning - Beiträge zur E-Didaktik und darüber hinaus“. Aarau, Sauerländer, 2003

Michael H. Breitner, Gabriela Hoppe (Hrdg.): „E-Learning-Einsatzkonzepte und Geschäftsmodelle“. Physica-Verlag, 2004

Michael Derntl, Renate Motschnig-Pitrik: „Pattern for Blended, Person-Centered Learning: Strategy, Concepts, Experiences and Evaluation". Technical Report No. TR-20033004, Department of Computer Science and Business Informatics, University of Vienna, Oktober 2003

Michael Derntl, Renate Motschnig-Pitrik: „The role of structure, patterns, and people in blended learning". Internet and Higher Education 8 (2005) 111-130

Werner Jank, Hilbert Meyer: „Didaktische Modelle”. Cornelsen Scriptor, 1991

MASGF (Ministerium für Arbeit, Soziales, Gesundheit und Familie) des Landes Brandenburg (Hg.): „Brandenburger Fachkräftestudie - Entwicklung der Fachkräftesituation und zusätzlicher Fachkräftebedarf". Potsdam, 2005

Helmut Niegemann u.a.: „Kompendium E-Learning. X.media.press“. Springer, 2004

Jan Pawlowski: „Das Essener-Lern-Modell (ELM): Ein Vorgehensmodell zur Entwicklung computerunterstützter Lernumgebungen“. Dissertation, Essen, 2001

\section{Autoren}

Prof. Dr. Ulrike Tippe

Technische Fachhochschule Wildau

Fachbereich Betriebswirtschaft/ Wirtschaftsinformatik

Tel. +49 3375 508-556

utippe@wi-bw.tfh-wildau.de

Prof. Dr. Bertil Haack

Technische Fachhochschule Wildau

Fachbereich Wirtschaft, Verwaltung und Recht

Tel. +49 3375 508-914

bhaack@wvr.tfh-wildau.de 